NOTE BREVI

\title{
Bi-Hamiltonian Structure of a Pair of Coupled KdV Equations.
}

\section{Y. NUTKU}

Department of Mathematics, Bilkent University - 06533 Bilkent, Ankara, Turkey

\section{Ö. OG̃UZ}

Department of Physics, Bog̃azi ci University - Bebek, Istanbul, Turkey

(ricevuto il 18 Giugno 1990)

Summary. - We point out a pair of coupled KdV equations which admits a bi-Hamiltonian structure.

PACS 02.30.Ks - Finite differences and functional equations.

The KdV equation is the example of a bi-Hamiltonian system[1]. There exists a number of generalizations of this equation which are interesting from the point of view of the Hamiltonian structure. In this connection the Hirota-Satsuma system [2] and its hierarchy [3] have been discussed by Oevel[4] and Aiyer [5]. Extensive discussions of the multi-Hamiltonian structure of various coupled $\mathrm{KdV}$ equations have been presented by Antonowicz and Fordy [6].

We shall point out a new system

$$
u_{t}=2 a u u_{x}+v v_{x}+(u v)_{x}+u_{x x x}, \quad v_{t}=2 b v v_{x}+u u_{x}+(u v)_{x}+v_{x x x},
$$

where $a, b$ are constants subject to

$$
a+b=1,
$$

which admits a bi-Hamiltonian structure. For $a= \pm b$ these equations decouple. We shall now discuss the Hamiltonian structure [7] of eqs. (1).

It is readily verified that the primary Hamiltonian structure of eqs. (1) is given by

$$
U_{t}=J_{0} E\left(H_{1}\right) \text {, }
$$

where $U$ is a 2-component field and $E$ denotes the corresponding Euler, or variational 
derivative

$$
U=\left(\begin{array}{l}
u \\
v
\end{array}\right), \quad E=\left(\begin{array}{c}
\frac{\delta}{\delta u} \\
\frac{\delta}{\delta v}
\end{array}\right),
$$

with the first Hamiltonian operator and function

$$
\begin{gathered}
J_{0}=\left(\begin{array}{cc}
D & 0 \\
0 & D
\end{array}\right), \quad D=\frac{\partial}{\partial x} ; \\
H_{1}=\frac{1}{3}\left(a u^{3}+b v^{3}\right)+\frac{1}{2}\left(u^{2} v+u v^{2}\right)-\frac{1}{2}\left(u_{x}^{2}+v_{x}^{2}\right),
\end{gathered}
$$

respectively. However, we note that in addition to $H_{1}$ eqs. (1) also admit

$$
H_{0}=\frac{1}{2}\left(u^{2}+v^{2}\right)
$$

as a conserved quantity. This suggests the existence of a second Hamiltonian operator $J_{1}$ satisfying the recursion relation

$$
J_{1} E\left(H_{k-1}\right)=J_{0} E\left(H_{k}\right),
$$$$
k=1,2,3 \ldots
$$

which will give rise to infinitely many conserved quantities $\left\{H_{k}\right\}$. The required second Hamiltonian operator is given by

$$
J_{1}=\left(\begin{array}{cc}
D^{3}+m D+D m & p D+D p \\
p D+D p & D^{3}+n D+D n
\end{array}\right)
$$

with

$$
m=\frac{1}{3}(2 a u+v), \quad n=\frac{1}{3}(u+2 b v), \quad p=\frac{1}{3}(u+v),
$$

and it can be easily verified that eq. (9) satisfies the Jacobi identities and is compatible with $J_{0}$. Thus the conditions of Magri's theorem are satisfied and we obtain an infinite set of conserved Hamiltonians which are in involution with respect to Poisson brackets defined by either one of these Hamiltonian operators. From the recursion relation (8) it follows that

$$
\begin{aligned}
H_{2}=\frac{5}{72}\left(1+4 a^{2}\right) u^{4}+\frac{5}{72}(1 & \left.+4 b^{2}\right) v^{4} \frac{5}{6} u^{2} v^{2}+ \\
& +\frac{5}{18}(1+2 a) u^{3} v+\frac{5}{18}(1+2 b) u v^{3}-\frac{5}{6}\left(u_{x}^{2} v+u v_{x}^{2}\right)- \\
& \quad-\frac{5}{3} u_{x} v_{x}(u+v)-\frac{5}{3}\left(a u u_{x}^{2}+b v v_{x}^{2}\right)+\frac{1}{2}\left(u_{x x}^{2}+v_{x x}^{2}\right)
\end{aligned}
$$

is the next conserved quantity in this sequence. 


\section{REFERENCES}

[1] F. MagrI: J. Math. Phys., 19, 1156 (1978).

[2] R. Hirota and J. Satsuma: Phys. Lett. A, 85, 407 (1981).

[3] D. LeVI: Phys. Lett. A, 95, 7 (1983).

[4] W. Oevel: Phys. Lett. A, 94, 404 (1983).

[5] R. N. AIYER: Phys. Lett. A, 93, 368 (1983).

[6] M. Antonowicz and A. P. FoRdy: Physica (Utrecht) D, 28, 345 (1986); Phys. Lett. A, 122, 95 (1987).

[7] P. J. OLver: Applications of Lie Groups to Differential Equations, Graduate Texts in Mathematics, Vol. 107 (Springer, New York, N.Y., 1986). 